\title{
Federation of Surgeons of South Africa consensus document for the resumption of elective surgery after level 5 COVID-19 "lockdown" period in South Africa
}

\author{
J van Waart, ${ }^{1}$ PJ Matley, ${ }^{2}$ M Brand ${ }^{3}$ on behalf of FoSAS \\ ${ }^{1}$ Private Practice, South Africa \\ ${ }^{2}$ Vascular Surgeon, South Africa \\ ${ }^{3}$ Hepatopancreaticobiliary Surgery, Steve Biko Academic Hospital, South Africa
}

The current document offers a set of principles and issues to help local facilities (with the guidance of the local COVID-19 committees) plan for resumption of elective surgical care.

\section{Introduction}

Since the introduction of a national state of disaster at the end of March 2020 we have moved through levels of "lockdown" and as of 1 June 2020 it has been de-escalated to level 3. It is unclear as to how long this level will remain in place. However, it is very likely that our healthcare infrastructure and resources, specifically with relation to the care of the most critically ill patients, will be strained over the coming weeks and months.

With the requirement for our profession to provide critical care services and the possibility that our operating theatres may be converted into intensive care units, it becomes our collective responsibility to take appropriate steps to support measures that will "flatten the curve". This is a dynamic document with its recommendation being effective at the time of issue and may be updated or changed at any time.

At this stage we do not propose a list of surgical procedures, but rather categories into which we encourage surgical/procedural teams to classify their patients. For the purposes of this document we define elective and other surgery/procedures as follows:

- Discretionary surgery is surgery that is scheduled in advance and where postponement of the surgery/ procedure will not result in the patient's outcome or quality of life being significantly altered with a three month delay.

- Essential surgery is a surgery that is scheduled in advance and where postponement of the surgery will result in the patient's outcome or quality of life being significantly altered if extended beyond three months.

- Urgent surgery is surgery that must be performed in order to preserve the patient's life or limb or prevent longer term systemic morbidity, but does not need to be performed immediately and should be performed within two weeks.
- Emergency surgery is surgery that must be performed without delay or when the patient is medically stable; the patient has no choice other than to undergo immediate surgery if permanent disability or death is to be avoided.

It is impossible to define the medical urgency of a case solely on whether a case is on an elective surgery schedule. While some cases may be postponed indefinitely, the majority of cases performed are associated with progressive diseases which, by their nature, will continue to progress at variable, disease-specific rates. Hence the judgement as to whether or not a surgical intervention should take place can only be decided once numerous considerations have been observed.

Given the uncertainties regarding the impact of COVID-19, delaying some cases risks having them reappear as emergencies at a time when they will be less easy to manage. FoSAS strongly urges communication and collaboration between hospital clinicians and their administrative staff to consider the following recommendations:

1. Hospitals and surgical teams should consider both their patient's medical needs and their logistical capacity to meet those needs in an appropriate time frame.

2. The medical need for a given procedure should be established by a surgeon with direct expertise in the relevant surgical speciality to determine which medical risks will be incurred by a delay.

3. Logistical feasibility for a specific procedure should be determined by management personnel with an understanding of hospital and community limitations. This includes facility resources (e.g. beds, staff, equipment etc.) as well as the safety and well-being of care providers and local community.

4. Case conduct should be determined based on knowledge of national, regional and local evolving conditions, recognising that marked regional variation may lead to significant differences in regional decision making. 
5. The risk to the patient should include an aggregate assessment of the real risk of proceeding and the real risk of delay, including the expectation that a delay of several weeks (or months) may be required to progress to an environment where COVID-19 is less prevalent.

In general, a day by day, evidence-driven assessment of the changing risk-benefit analysis will need to influence clinical care decisions and delivery for the foreseeable future. Plans for case triage should avoid blanket policies and instead rely on data and expert opinion from qualified (and well informed) clinicians and management teams understanding all COVID-19 issues in play. Although COVID-19 is a clear risk to all, it is but one of many competing risks for patients requiring surgical care. Thus, surgical procedures should be considered not based solely on COVID-19-associated risks, but rather on an assimilation of all available medical and logistical information.

\section{Diagnostic testing}

As the anaesthetists are the healthcare workers (HCW) with the highest risk of infection in the surgical team, SASA guidelines in testing patients preoperatively should be carefully considered in local policy. (This FoSAS document should be read, taking the SASA document "A pragmatic approach to surgery during COVID-19 period in South Africa" into consideration).

Know your COVID-19 diagnostic testing availability, and develop operational testing policies for patients and healthcare workers:

- Know, understand, and update your local COVID-19 diagnostic testing capabilities and turnaround times. The testing availability will likely change during the ramp-up period. While it is to be hoped that availability is on the rise, some predict that availability may actually decrease as the community testing demands increase.

- Develop local diagnostic testing policies for patients. Rapid testing for COVID-19 infection, through realtime reverse transcription polymerase chain reaction (RT-PCR) testing, may be considered for all patients undergoing planned surgery, or for selected patients after screening with or without mandatory preoperative quarantine. The prevalence of asymptomatic/presymptomatic patients is unknown, but likely varies according to the pre-test probability, i.e. prevalence of disease in the community. Surgeons should be involved in institutional policy-making since the risk to the patient and the staff varies with the type of procedure, the patient's condition, local circumstances, and over time. Some surgeon discretion is necessary and should be permitted.

- Develop diagnostic screening testing policies for healthcare workers. With near-future reversal of physical distancing, local incidence may increase, including among healthcare workers. As ramp-up proceeds, screening and testing policies and planning for staff should be considered.

- Consider false negative test rates and need for retesting. False negatives have been reported as high as 30 per cent. Guidelines for potential retesting in negative patients might be considered. A particular challenge to healthcare worker safety is our current lack of understanding of duration for transmissibility of the virus in either asymptomatic COVID-19-positive patients or individuals who have recovered from a COVID-19 illness. There is evidence that even after respiratory samples are negative in patients who have recovered from a COVID-19 illness, viral RNA remains in the stool for $>30$ days. The clinical significance of faecal RNA is not well understood.

- Consider guidelines for postoperative COVID-19 testing of symptomatic patients/patients under investigation (PUI). Atelectasis, fevers, etc., are not uncommon in the postoperative course. Establishing operational guidelines for COVID-19 testing in these patients and concurrent testing results should be considered.

- There is not likely to be a highly sensitive and specific mass testing ability for at least several months. Therefore, reasonable alternative methods of determining risk versus benefit to the patient and public health in all facilities, inpatient and outpatient, will be required in the interim in order to continue the care of patients now waiting for surgeries previously delayed during the first phase of the pandemic. If optimal screening/testing is unavailable locally, implementation of such alternative screening methods is a local decision.

Ensure safe, high-quality, high-value care of the surgical patient across the five phases of care continuum.

Informed consent from patients should not only include procedure-related risks, but also COVID-19-associated risks (e.g. chance of contracting the disease in hospital etc.). This consent should be done in writing and ideally by the surgeon involved.

\section{Across the five phases of care}

- Utilise quality improvement programmes/care standards to help support achieving safe, high-quality, high-value patient care.

- Use of risk-adjusted data to evaluate patient care and outcomes.

- Ensure optimal patient care.

\section{Phase I: Preoperative period}

- Consider guideline for repeating laboratory results, radiology, history and physical, re-consent vs use of prior results. Consider use of telehealth.

- (Also a good idea to advise patients to have the flu vaccine, as RSA is entering the flu season.)

- Consider guideline to (re)assess comorbidities especially if COVID-19/PUI or extended length of time of postponed operation. Consider use of telehealth.

- The composite assessment, in conjunction with sound clinical judgment, provides the surgeon and other decision makers with the information needed to make decisions regarding clinical appropriateness as well as surgical prioritisation.

- $\quad$ Office, clinic, hospital public areas (e.g. waiting room) should continue to practise physical distancing (e.g. 1.5 metre spacing of chairs).

- Consider review of patient advance directive, especially older adults, frail, COVID-19+, other.

- Evaluate and discuss patient's potential need for postacute care facility (rehabilitation medicine, skilled nursing facility, other) before operation (given known COVID-19 outbreaks in post-acute care-type facilities).

- Preoperative setting (e.g. clinic, office, or nonCOVID-19 care [NCC] areas) should consider 
screening all patients before the appointment for symptoms of COVID-19 disease, including temperature checks, and routinely screen all staff and others who would be working in the facility (physicians, nurses, housekeeping, delivery, and others).

- Consider a policy for all healthcare providers and staff to wear surgical face masks at all times. Procedures on the mucous membranes, including the respiratory tract, that have a higher risk of aerosol transmission should be done with great caution, and staff should utilise appropriate respiratory protection such as N95 masks and face shields.

- All patients should wear a cloth face mask that can be bought or made at home.

\section{Phase II: Immediate preoperative period}

- Review existing nursing, anaesthesia and surgery checklists for the need of additional COVID-19 precautions.

\section{Phase III: Intraoperative period}

- Review whether time-outs and briefings need revision with regards to COVID-19 risk, COVID-19 testing results, and ensure PPE use guidelines are being followed.
- Waiting time (e.g. with regards to air circulation cycling time) before beginning operation.

- $\quad$ PPE use guideline (see above).

- Review specimen pick-up protocol.

\section{Phase IV: Postoperative period}

- Adhere to standardised care protocols as much as possible (e.g. enhanced recovery protocols) for increased reliability in light of potential different personnel as standardised protocols optimise lengths of hospital stay and efficiency and are associated with decreased complication rates.

\section{Phase V: Post-discharge period}

- Post-acute care facility availability.

- Post-acute care facility safety (COVID-19, nonCOVID-19 issues).

- Home setting.

In conclusion, sensible communication is essential between clinicians, their associated healthcare workers and hospital administrators to determine the most appropriate clinical care paths for all patients. It is in the interests of all healthcare workers and administrators to work together to ensure safety as well as sustainable practice.

Note: At the time of issue of this document, Stage 3 "lockdown" government documentation seems to allow for (all) medical services to continue. (The extent of services needs to be confirmed with the Department of Health.) FoSAS reiterates that (if consultations resume) all measures to safeguard against the spread of the COVID-19 virus (screening processes, hand hygiene, wearing of appropriate masks, social distancing etc.) should be in place in areas where patients gather.

Note: This is not a prescriptive, binding document and in no way provides medicolegal coverage. All healthcare workers must still act in a reasonable manner which is justifiable under the circumstances in which they work.

\section{FEDERATION OF SOUTH AFRICAN SURGEONS FoSAS}

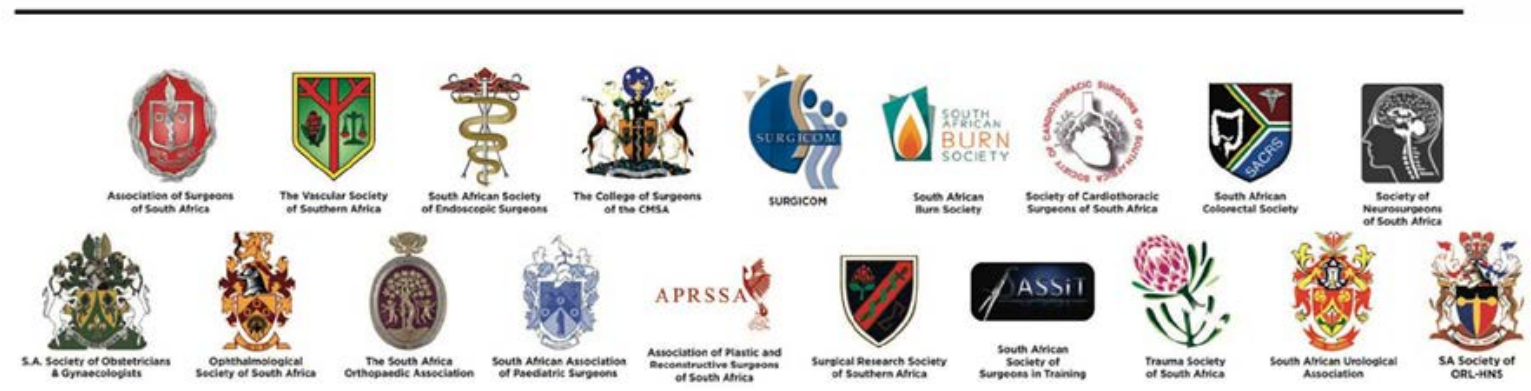

\section{KEY REFERENCES}

- SASA Position Statement - A pragmatic approach to surgery after the "lockdown" period in South Africa. April 2020. Available from: https://sasacovid19.com. Accesssed 15 April 2020.

- Joint Statement: Roadmap for resuming elective surgery after COVID-19 Pandemic. American College of Surgeons, American Society of Anesthesiologists, Association of Perioperative Registered Nurses, American Hospital Association. 17 April 2020. Available from: https://www. asahq.org/about-asa/newsroom/news-releases/2020/04/joint- statement-on-elective-surgery-after-covid-19-pandemic. Accessed 15 April 2020.

- COVID-19: Guidance for triage of non-emergent surgical procedures. American College of Surgeons, 17 March 2020. Available from: https://www.facs.org/covid-19/clinicalguidance/triage. Accessed 15 April 2020.

- COVID-19: Elective case triage; guidelines for surgical care (PDF). American College of Surgeons, 27 March 2020. Available from: https://www.facs.org/-/media/files/covid19/ guidance_for_triage_of_nonemergent_surgical_procedures. ashx 